\title{
EL CONSENTIMIENTO HIPOTÉTICO DE LA VÍCTIMA EN EL DERECHO PENAL ALEMÁN*
}

\section{ANDRÉS SCHLACK M. ${ }^{* *}$}

RESUMEN: El presente artículo examina la figura jurídica del así denominado "consentimiento hipotético" de la víctima, introducida en la jurisprudencia penal reciente del Tribunal Supremo Federal alemán (BGH) y que ha recibido aplicación en casos de intervenciones médicas con fines curativos realizadas sin el consentimiento informado del paciente. En tales casos, el BGH ha negado la responsabilidad penal del médico por el delito de lesiones, de comprobarse que el paciente, de haber sido debidamente informado, hubiera consentido previamente en la intervención realizada. Se examina asimismo la recepción de esta figura por una parte de la doctrina alemana y se exponen finalmente los fundamentos que, a juicio del autor, deben conducir a su rechazo.

PALABRAS CLAVE: Consentimiento hipotético - actividad médica imputación objetiva - autonomía del paciente - consentimiento presunto.

\section{HYPOTHETICAL CONSENT OF THE VICTIM IN GERMAN CRIMINAL LAW}

ABSTRACT: This article examines the legal concept of the so-called "hypothetical consent" of the victim, introduced in the recent criminal jurisprudence of the German Federal Supreme Court (BGH) and it has received application in cases of medical interventions with healing purposes made without the informed consent of the patient. In such cases, the BGH has denied criminal liability of the doctor for the crime of medical injury, after probing that the patient was duly informed and the patient had previously consented to the intervention. It also examines the reception of this figure as part of the German doctrine and finally the fundaments that lead to its rejection, according to the author's judgments.

\footnotetext{
* Fecha de recepción:17 de febrero de 2012.

Fecha de aceptación: 21 de agosto de 2012.

** Abogado. Licenciado en Derecho por la Pontificia Universidad Católica de Chile. Magister Legum (LL.M.) por la Rheinische Friedrich-Wilhelms-Universität Bonn. Doctorando en Derecho en el Instituto de Derecho Penal de la Rheinische Friedrich-Wilhelms-Universität Bonn. Becario de la Konrad-Adenauer-Stiftung. Dirección de correo electrónico: aeschlack@gmail.com.
} 
KEY WORDS: Hypothetical consent - medical activity - objective imputation - patient autonomy - presumed consent.

\section{1) EL CONCEPTO DEL CONSENTIMIENTO HIPOTÉTICO}

El concepto del consentimiento hipotético, desarrollado en la jurisprudencia del Tribunal Supremo Federal de Alemania (BGH), se refiere a casos de incumplimiento de deberes de información en el ámbito de intervenciones médicas curativas. Como consecuencia de dicho incumplimiento, el consentimiento de la víctima respecto de la acción típica ${ }^{1}$ conforme al $\$ 223$ StGB (tipo básico de lesiones) resulta inválido ${ }^{2}$. Un consentimiento presunto de la víctima con efectos justificantes también debe de ordinario descartarse, por cuanto falta por regla general el requisito esencial relativo a la imposibilidad de obtener oportunamente el consentimiento expreso de la víctima debido a obstáculos insuperables ${ }^{3}$.

De acuerdo con la postura del BGH, solamente puede afirmarse en este supuesto la punibilidad de la conducta del médico por lesiones en aquellos casos en que el paciente, debidamente informado, no hubiera consentido en la intervención realizada ${ }^{4}$. Cuando no pueda excluirse que el paciente hubiera prestado su consentimiento en caso de haber sido informado suficientemente, se debe en conformidad al principio in dubio pro reo afirmar en favor del médico la presunción de que la víctima hubiera consentido en la intervención. ${ }^{5}$

1 Desde RGSt 25, 375 y de acuerdo a una jurisprudencia sostenida, las intervenciones médicas curativas deben ser subsumidas como lesiones típicas, las que pueden ser justificadas mediante el consentimiento del paciente. Frente a esta postura de la jurisprudencia, en la literatura impera la denominada teoría del resultado (Erfolgstheorie), de acuerdo a la cual debe distinguirse entre intervenciones curativas exitosas y fallidas. En el caso de las primeras se excluye la tipicidad. Sobre la corrección de esta tesis de la jurisprudencia alemana no nos es posible tomar partido aquí. Para una visión de conjunto sobre este punto, véase KINDHÄUSER, Urs (2012). Strafrecht Besonderer Teil I. 5a edición. Baden-Baden: Nomos, \$8 / NM 22 ss.; Tröndle, Herbert (1983). “Selbsbestimmung des Patienten - Wohltat und Plage?”. MDR 1983, pp. 881-887.

2 Bollacher, Florian / Stockburger, Jochen (2006). "Der ärztliche Heileingriff in der strafrechtlichen Fallbearbeitung”. Jura 2006, pp. 908-914, p. 911. Para las distintas opiniones concernientes al tratamiento del consentimiento basado en un error y, en especial, del tratamiento del consentimiento obtenido mediante engańo, Oтто, Harro (2004). "Einwilligung, mutmaßliche, gemutmaßte und hypothetische Einwilligung”. Jura 2004, pp. 679683, p. 680.

3 KindhäUser, Urs (2011). Strafrecht Allgemeiner Teil. 5a edición. Baden-Baden: Nomos, $\$ 19 /$ NM 15.

5 BGH NStZ 1996, 35; BGH JR 2004, 252; BGH NStZ 2012, 206. 


\section{2) EL CONSENTIMIENTO HIPOTÉTICO EN LA JURISPRUDENCIA PENAL DEL BGH}

Con el reconocimiento de un efecto excluyente de la responsabilidad penal del consentimiento hipotético por parte del $\mathrm{BGH}^{6}$ se trasladó a la jurisprudencia penal de dicho tribunal una figura jurídica desarrollada originalmente en la jurisprudencia civil ${ }^{7}$. Según la doctrina del $\mathrm{BGH}$, tiene el consentimiento hipotético por consecuencia que la antijuricidad de la conducta decae ${ }^{8}$. Sin embargo, el BGH no proporciona ninguna fundamentación dogmática exhaustiva de dicho efecto excluyente de la antijuricidad.

En los siguientes casos desempeña el consentimiento hipotético un papel fundamental:

\section{1) BGH NSTZ 1996, 349}

En esta sentencia, el supuesto de hecho se refiere al incumplimiento del deber de informar respecto de varios pacientes sobre las diversas posibilidades existentes respecto de materiales para implantes óseos y sobre las ventajas o desventajas y los riesgos concretos de complicaciones del material efectivamente usado. A este respecto manifestó el BGH que "la falta de información puede (...) fundamentar una punibilidad del médico por lesiones solamente cuando el paciente, de haber sido debidamente informado, no hubiera consentido en la intervención (...). Esto debe probarse en contra del médico. Si permanecen dudas, debe partirse de la base que el consentimiento hubiera sido prestado en caso de una información conforme a deber" 10 .

Además, según la opinión del BGH, no todo déficit de información debida conduce a una punibilidad por lesiones. Bajo el punto de vista del

6 Ya en BGHR StGB $\$ 223$ I Heileingriff 2, 1, 3. Véase el comentario a esta sentencia de PupPE, Ingeborg (2011). Strafrecht Allgemeiner Teil im Spiegel der Rechtsprechung. 2a edición. Baden-Baden: Nomos, $\$ 11$ / NM 18 .

7 Sobre el desarrollo del consentimiento hipotético en la jurisprudencia civil véase SICKOR, Jens Andreas (2008). "Die übertragung der hypothetischen Einwilligung auf das Strafrecht". JA 2008, pp. 11-16, pp. 12 ss. [en adelante, citado como Sickor (2008a)].

$8 \quad$ BGH JR 2004, 252; BGH NStZ 2004, 442; BGH NStZ-RR 2007, 341.

9 Sentencia del BGH de 29.6.1995 - 4 StR 760 / 94 = JR 1996, 69. Comentario de UlsenHeimer, Klaus (2008). NStZ 1996, pp. 132-133, p. 132 ss.; Puppe, Ingeborg (2003). "Die Strafrechtliche Verantwortlichkeit des Arztes bei mangelnder Aufklärung über eine Behandlungsalternative“. GA 2003, 764-776; RigizaHn, Ernest (1996). JR 1996, pp. 72-75, pp. 72 ss.; Kunlen, Lothar (2001). "Objektive Zurechnung bei Rechtfertigungsgründen”. En Schünemann, Bernd, et al. (coordinadores): Festschrift für Klaus Roxin zum 70. Geburtstag am 15. Mai 2001. Berlín-Nueva York: Walter de Gruyter, pp. 331-347, pp. 341 ss. [en adelante, citado como KuHLen (2001a)].

10 BGH NStZ 1996, 35. Aquí y en adelante, todas las traducciones de citas de jurisprudencia y de doctrina desde el idioma alemán son del autor. 
fin de protección de la norma, la punibilidad de un médico por la falta de cumplimiento de su deber de informar resulta excluida "cuando como consecuencia de una intervención realizada con el consentimiento del paciente se realiza un riesgo que no se encuentra en el ámbito de protección del deber de información infringido" 11 .

\section{2) BGH JR 2004, $251^{12}$}

Con este fallo encontró el consentimiento hipotético aplicación no solamente en supuestos de error imprudente en el cumplimiento del deber de informar, sino también en casos de déficit del consentimiento basados en un engaño por parte del médico. Los hechos se refieren al ocultamiento de los verdaderos motivos de una segunda intervención médica destinada a reparar un error médico cometido en una primera intervención y fundamentada ante el paciente con un motivo conocidamente falso.

Respecto del consentimiento hipotético, señaló el BGH: "La antijuricidad decae, sin embargo, cuando el paciente en caso de haber informado conforme a la verdad hubiera consentido en la intervención efectivamente realizada" 13 . Con ello se radica la prueba del consentimiento hipotético en el ámbito de la antijuricidad.

La decisión del BGH sugiere, sin embargo, un entendimiento causal del problema del déficit en el deber de informar ${ }^{14}$ y propone una fórmula individual para la comprobación del consentimiento hipotético: "En la prueba de causalidad se debe estar al resultado concreto de la decisión de un determinado paciente. No se trata de que hubiera consentido en todo caso en la operación o que un paciente razonable hubiera dado su consentimiento (...). Es insuficiente que la segunda intervención en cuanto al resultado se adecue a su voluntad e interés" 15 .

\section{3) BGH NSTZ 2004, $442^{16}$}

En este caso se fingió por parte de un médico ante el paciente una indicación médica falsa para una segunda intervención, cuyo verdadero

11 BGH NStZ 1996, 35.

12 Sentencia del BGH de 15.10.2003 - 1 StR $300 / 03=$ BGH NStZ-RR 2004, 16 = BGH JZ 2004, 800, con comentario de RönnaU. Véase también comentarios de KuHLEN, Lothar (2004). "Ausschluss der objektiven Erfolgszurechnung bei hypothetischer Einwilligung des Betroffenen”. JR 2004, pp. 227-230, p. 227, y Eisele, Jörg (2005). "Hypothetische Einwilligung bei ärtzlichen Aufklärungsfehlern”. JA 2005, pp. 252-254, p. 252.

BGH JR 2004, 252.

Véase sobre este punto infra 4.5.1.

BGH JR 2004, 252.

Sentencia del BGH de 20.01.2004 - 1 StR 319 / 03 = BGH JR 2004, 469, con comentario de Puppe. 
motivo era la extracción de una punta de taladro que se rompió y se incrustó en un hueso del paciente durante una primera intervención. En la sentencia confirmó el $\mathrm{BGH}$ un fallo condenatorio de primera instancia en contra del médico: "en base a la determinación inequívoca de que el paciente E no hubiera consentido en la extracción de la punta de taladro quebrada, lo que no deja espacio a la suposición, de que la antijuricidad podría decaer por el hecho de haber sido conducida la operación según la lex artis (...)"17. Pese a que la decisión del BGH negó el consentimiento hipotético en este caso concreto, se reconoció en general el efecto excluyente de la antijuricidad de dicho consentimiento cuando concurre.

\section{4) BGH NSTZ-RR 2007, $340^{18}$}

En esta resolución, referida a un segundo procedimiento de liposucción realizado respecto de un paciente y que condujo a la muerte de este, limitó el BGH la aplicación del consentimiento hipotético exclusivamente a intervenciones realizadas conforme a la lex artis: "el consentimiento en una intervención médica curativa, al menos en caso de falta de una información conforme a deber, se refiere solamente a un tratamiento curativo realizado en conformidad con la lex artis, es decir, con el estado actual de la ciencia médica"19. Decisivo resulta solamente "si A, también en conocimiento de las mencionadas circunstancias, diversas de aquellas de la primera operación, hubiera consentido en la intervención" 20 .

\section{3) LA UBICACIÓN SISTEMÁTICA DEL CONSENTIMIENTO HIPOTÉTICO EN LA DOCTRINA ALEMANA}

El consentimiento hipotético ha encontrado apoyo en una parte de la doctrina alemana ${ }^{21}$. Sin embargo, es debatido por aquellos autores par-

17 BGH NStZ 2004, 442.

18 Sentencia del BGH de 05.07.2007 - 4 StR 549/06 = BGH StV 2008, 189, con comentario de Sternberg-Lieben. Véase también comentario de Bosch, Nikolaus (2008). "Grenzen einer hypothetischen Einwilligung bei ärtzlichem Eingriff'. JA 2008, pp. 70-72.

19 BGH NStZ-RR 2007, 341. Interesante resulta además que el BGH impuso exigencias más estrictas en este caso respecto del deber de informar, pues no se trataba en este caso de una intervención propiamente curativa, sino de un tratamiento cosmético, que no era urgente ni estaba médicamente indicado.

20 BGH NStZ-RR 2007, 341.

21 Kuhlen (2001a) 331; Kuhlen (2001). "Ausschluß der objektiven Zurechnung bei Mängeln der wirklichen und der mutmaßlichen Einwilligung”. En BRITZ, Guido, et al. (coordinadores): Grundfragen staatlichen Strafens: Festschrift für Heinz Müller-Dietz zum 70. Geburtstag. München: Beck, pp. 431-451, p. 431 [en adelante, citado como KuHLEN (2001b)]; Kuhlen (2004) 227; Kuhlen, Lothar (2005). "Hypothetische Einwilligung und Erfolgsrechtfertigung”. JZ 2005, pp. 713-718, p. 713; Mrtsch, Wolfgang (2005). “Die 
tidarios de esta figura cómo debe ser clasificada desde un punto de vista sistemático. A grandes rasgos, es posible encontrar entre los defensores del consentimiento hipotético tres modelos de fundamentación distintos, los que seguidamente se exponen.

\section{1) KUHLEN: DESPLAZAMIENTO DE LOS CRITERIOS DE IMPUTACIÓN OBJETIVA AL PLANO DE LA ANTIJURICIDAD}

Kuhlen postula una fundamentación del consentimiento hipotético mediante el desplazamiento de la imputación objetiva del plano de la tipicidad al ámbito de la antijuricidad ${ }^{22}$. Según Kuhlen, no corresponde el consentimiento hipotético a una causal de justificación, sino una causal de exclusión de punibilidad (Strafbarkeitsausschluss), la cual determina la negación de la imputación objetiva ante un supuesto en que faltan los requisitos de la justificante ${ }^{23}$.

El autor ve una analogía estructural entre el consentimiento hipotético y la causal de exclusión de la imputación objetiva reconocida en el plano de la tipicidad (en el ámbito del delito culposo) relativa a la falta de un nexo de infracción de un deber (Pflichtwidrigkeitszusammenhang). Los elementos del nexo de infracción de deber y de la realización de un riesgo específico creado (Risikozusammenhang) respecto de la infracción del deber de información, por una parte, y su relación con el resultado, por otra, deben ser probados según Kuhlen también en el nivel de la antijuricidad objetiva, y la falta de dichos elementos excluye el injusto objetivo de un delito consumado 24 .

Luego de la comprobación de que no concurren los requisitos de ninguna causal de justificación, debe todavía probarse en el plano de la antijuricidad objetiva si el resultado descansa objetivamente en la falta de realización de una causal de justificación ${ }^{25}$. La falta de cada uno de los requisitos de una causal de justificación respecto de una conducta típica es insuficiente para excluir el injusto de un delito consumado ${ }^{26}$. Según la postura de Kuhlen, los criterios de imputación objetiva deben

hypothetische Einwilligung im Arztstrafrecht". JZ 2005, pp. 279-285, p. 279; RigizaHN (1996) 72; Rönnau, Thomas (2004). Anmerkung zu BGH JZ 2004, 800. JZ 2004, pp. 801-804, p. 801; Roxin, Claus (2006). Strafrecht Allgemeiner Teil I: Grundlagen- Der Aufbau der Verbrechenslehre”. 4a edición. München: Beck, $\$ 13$ / NM 118 ss.; Rosenau, Henning (2010). "Die hypothetische Einwilligung im Strafrecht". En Bloy, René, et al. (coordinadores): Gerechte Strafe und legitimes Strafrecht, Festschrift für Manfred Maiwald zum 75. Geburtstag. Berlin: Duncker \& Humblot, pp. 683-700.

Kuhlen (2001a) 332; Kuhlen (2001b) 432; Kuhlen (2004) 227; Kuhlen (2005) 713.

Kuhlen (2001a) 334; Kuhlen (2004) 227.

KuHLen (2001a) 338.

Kuhlen (2001a) 339; Kuhlen (2001b) 432; Kuhlen (2005) 716.

Kuhlen (2001a) 339; Kuhlen (2001b) 433. 
ser considerados en el plano de la antijuricidad solamente cuando el cumplimiento de la causal de justificación en su faz objetiva es excluido por lo que llama un "mero déficit de justificación" (bloße Rechtfertigungsmangel), sobre el cual el resultado típico debe descansar y serle objetivamente imputable. ${ }^{27}$ En la formulación de Kuhlen, tiene la doctrina de la imputación objetiva en casos de no concurrencia de una causal de justificación la tarea de distinguir entre déficits irrelevantes para efectos de la imputación de aquellos que sí resultan relevantes en dicho plano ${ }^{28}$.

Según esta postura, el resultado no es objetivamente imputable al déficit del consentimiento cuando "el resultado también sin el déficit en el consentimiento (...) se hubiera producido igualmente (falta de un nexo de incumplimiento de deber) o cuando en el resultado no se ha realizado el riesgo específico del déficit de consentimiento (falta de realización del riesgo creado $)^{29}$. El nexo de incumplimiento de deber no está presente en casos de consentimiento hipotético cuando el paciente, en caso de haberse cumplido el deber de información, hubiera consentido en la operación ${ }^{30}$. No se realiza el riesgo específico creado cuando en la lesión producida no se realiza el riesgo específico creado por el déficit en la información, sino otro riesgo respecto del cual el paciente estaba debidamente informado o respecto del cual no existía deber de informar ${ }^{31}$.

La punibilidad del médico se encuentra excluida según Kuhlen en aquellos casos en que no resulte claro si el paciente, en caso de haber sido debidamente informado, hubiera entregado o no su consentimiento ${ }^{32}$. La posibilidad abstracta de que ante el cumplimiento del deber de informar el paciente pudiera haber consentido en la intervención curativa es insuficiente para excluir la punibilidad del médico ${ }^{33}$. Consideraciones concretas que se desprendan de los intereses o de la declaración de voluntad del paciente son necesarias para impedir una convicción del juez en los términos del $\$ 261$ StPO (el cual establece un estándar de libre valoración de la prueba para alcanzar el convencimiento judicial), de acuerdo con el principio in dubio pro reo ${ }^{34}$.

El consentimiento hipotético debe acreditarse mediante una consideración ex post. Una circunstancia que no era reconocible de manera

KuHLen (2001a) 339.

28 Kuhlen (2001b) 434. Según el autor los criterios de imputación objetiva deben tenerse en cuenta no solamente respecto del consentimiento (entendiendo dicho autor esta institución, junto con la doctrina aún dominante, como una causal de justificación), sino también respecto de todas las causales de justificación.

29 Kuhlen (2001a) 340; Kuhlen (2001b) 434.

30 Kuhlen (2001a) 342.

Kuhlen (2001b) 436.

Kuhlen (2001b) 435.

KuHLen (2001b) 435.

Kuhlen (2001b) 435; Kuhlen (2004) 229. 
previa a la intervención puede por consiguiente excluir el consentimiento hipotético, como por ejemplo un rechazo categórico del paciente a las transfusiones sanguíneas que solamente de manera posterior a la intervención llega a ser conocido ${ }^{35}$. Sin embargo, precisa Kuhlen que, a partir de un entendimiento del consentimiento hipotético orientado a la decisión del paciente, no resulta decisivo el resultado curativo, sino exclusivamente si el paciente debidamente informado y en desconocimiento del resultado de la operación hubiera prestado o no su consentimiento ${ }^{36}$.

Según la postura defendida por Kuhlen, el consentimiento hipotético de la víctima excluye la punibilidad del médico a título de delito consumado, pero considera posible la sanción a título de tentativa ${ }^{37}$. El que un delito tentado concurra o no dependerá decisivamente de la resolución de cometer un delito (dolo de consumación) del agente, la que estará presente cuando el autor no parte de la base de un consentimiento hipotético del paciente ${ }^{38}$.

Kuhlen le asigna una gran importancia a la posibilidad de una sanción a título de tentativa, en cuanto de ese modo es posible evitar a su juicio una equiparación del consentimiento hipotético con la causal de justificación del consentimiento presunto, equiparación que tornaría a esta última irrelevante ${ }^{39}$. Asimismo, le otorga al médico un motivo para prestar atención a la voluntad efectiva del paciente ${ }^{40}$.

\section{2) ROXIN: EL CONSENTIMIENTO HIPOTÉTICO Y LA TEORÍA DEL IN- CREMENTO DEL RIESGO}

De acuerdo con Roxin, el consentimiento hipotético excluye no la antijuricidad de la intervención, sino la imputación objetiva en el plano del tipo de lesiones ${ }^{41}$. El médico en los casos de consentimiento hipotético infringe su deber de información de un modo antijurídico y falta solamente la imputación objetiva del resultado, lo cual en caso de una comisión dolosa deja abierta la posibilidad de una sanción a título de tentativa ${ }^{42}$. Esta fundamentación sistemática del consentimiento hipotético

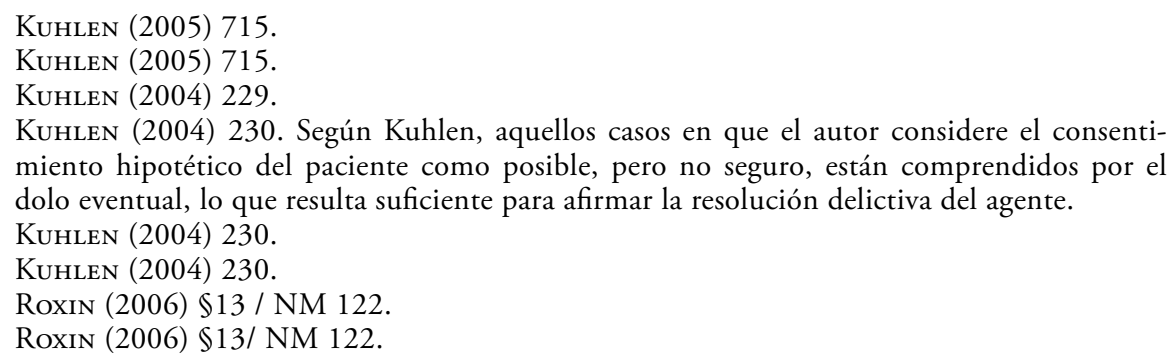


es consecuente con la comprensión de Roxin del consentimiento como un supuesto de exclusión de la tipicidad ${ }^{43}$.

Sin embargo, Roxin se manifiesta de un modo crítico respecto de la exigencia de la jurisprudencia relativa a la necesidad de prueba de que en caso de haberse dado cumplimiento al deber del médico de informar el consentimiento del titular del derecho no hubiera sido dado por este. En conexión con la teoría del incremento del riesgo (Risikoerhöhungslehre) ${ }^{44}$, defendida por este autor, debe afirmarse también la punibilidad de la conducta del médico en casos de falta de cumplimiento del deber de informar cuando exista la posibilidad que la víctima, en caso de haber sido debidamente informada, no hubiera prestado su consentimiento respecto de la intervención, ello por cuanto el riesgo típico se ha visto aumentado por la conducta del autor ${ }^{45}$. Debe descartarse la imputación del resultado típico debido a la irrelevancia del incumplimiento del deber de informar solamente cuando el paciente, de acuerdo con una consideración ex post, hubiera consentido con seguridad en la intervención en caso de haber sido debidamente informado ${ }^{46}$.

Para el enjuiciamiento ex post del consentimiento hipotético considera Roxin relevante la consulta al paciente con posterioridad al procedimiento médico, así como el contexto en que tuvo lugar la intervención (como, por ejemplo, si esta se encuentra médicamente indicada y no existe otra alternativa $)^{47}$.

\section{3) EL CONSENTIMIENTO HIPOTÉTICO COMO FALTA DE DISVALOR DE RESULTADO RELEVANTE SEGÚN MITSCH Y RÖNNAU}

Mitsch comprende el consentimiento hipotético como la falta de una lesión del bien jurídico en el sentido del $\$ 223$ StGB. Según este punto de vista, la información que el paciente recibe del médico tiene por fin protegerlo de una operación que implique una lesión al bien jurídico protegido por el $\$ 223 \mathrm{StGB}^{48}$. El que la intervención curativa sea o no una lesión de dicho bien jurídico puede ser determinado solamente mediante el resultado de la acción del agente: si se trata de un resultado que el titular del bien jurídico aprueba ex post de un modo tan intenso que hubiera consentido en la intervención, la acción médica carece entonces de la cualidad de una lesión en el sentido del $\$ 223 \mathrm{StGB}^{49}$. Sin un tipo penal

\footnotetext{
Roxin (2006) \$13/ NM 12 ss.

Roxin (2006) \$11/ NM 88 ss.

Roxin (2006) \$13/NM 124.

Roxin (2006) \$13/ NM 125.

Roxin (2006) \$13/NM 127.

Mitsch (2005) 285.

Mitsch (2005) 285.
} 
especial de acciones curativas no consentidas, la sola lesión del derecho de autodeterminación del paciente no puede fundar la punibilidad del médi$\mathrm{Co}^{50}$.

Según la opinión defendida por Mitsch, es el consentimiento hipotético un caso de cumplimiento parcial de una causal de justificación ("fragmentos de justificación”), el consentimiento presunto, lo que conduce a que el injusto de consumación resulte excluido ${ }^{51}$.

Este cumplimiento parcial se refiere a elementos objetivos de una causal de justificación, a través de los cuales se suprime el disvalor de resultado $^{52}$.

Sin embargo, el modo en que Mitsch comprende el enjuiciamiento ex post del consentimiento hipotético se aparta de la jurisprudencia y de los demás partidarios de esta figura jurídica ${ }^{53}$.

Según Mitsch, el consentimiento hipotético implica un titular del bien jurídico que en conocimiento del resultado efectivo de la intervención hubiera consentido en esta. Con palabras de Mitsch: "El titular del bien jurídico ficticio mira retrospectivamente en conocimiento del resultado y dice 'en razón de este resultado hubiera consentido / no hubiera consentido en el hecho"'54.

En términos similares, para Rönnau no concurre en el caso del consentimiento hipotético un disvalor de resultado: "El resultado producido no puede valorativamente calificarse como un resultado de injusto penalmente relevante, por cuanto el titular del bien jurídico hubiera consentido en la intervención de todos modos" 55 . Solamente una punibilidad a título de tentativa resultaría posible ${ }^{56}$.

\section{4) TOMA DE POSICIÓN: UNA CRÍTICA AL CONSENTIMIENTO HIPOTÉ- TICO}

El reconocimiento del consentimiento hipotético no está libre de considerables problemas dogmáticos, político-criminales y prácticos. Di-

Mitsch (2005) 285. De aceptarse esta opinión de Mitsch, distinto sería el caso en ordenamientos jurídicos como el austríaco, donde sí existe un tipo penal de tratamiento médico no consentido ( $\$ 110$ öStGB).

51 Mitsch (2005) 284. La punibilidad a título de tentativa, en cambio, es considerada por Mitsch como posible.

52 Mitsch (2005) 284. Ese es para Miтsch (2005) 283, el caso cuando una intervención curativa ha elevado las posibilidades de sanación del paciente. Con ello se tiene un resultado que hubiera sido suficiente para afirmar la justificación por consentimiento presunto, de haberse dado los restantes requisitos de esta figura.

53 Véase la valoración crítica de esta tesis por Kunlen (2005) 715 ss.

54 Miтsch (2005) 281.

55 Rönnau (2004) 802.

56 Rönnau (2004) 802. 
versos argumentos pueden esgrimirse en contra de esta figura jurídica, lo que conduce a que el consentimiento hipotético de la víctima no pueda ser aceptado como una causal de exclusión de la responsabilidad penal.

\section{1) IMPOSIBILIDAD DE UNA DETERMINACIÓN EX POST DEL CON- SENTIMIENTO HIPOTÉTICO DE LA VÍCTIMA}

Como ya ha argumentado Puppe ${ }^{57}$ en contra de los partidarios del consentimiento hipotético, no existe un método según reglas propias de las ciencias naturales para determinar cómo hubiera decidido el paciente si el médico hubiera cumplido con su deber de informar. De un modo diverso a aquellos procesos que son enteramente determinados por leyes naturales, las decisiones humanas libres no se dejan determinar causalmente por semejantes leyes y, por consiguiente, no puede responderse la pregunta de cómo alguien se hubiera comportado si las circunstancias hubieran sido distintas a la realidad. ${ }^{58}$ El paciente mismo no podría con certeza absoluta determinar si hubiera o no consentido en la intervención curativa de haber sido debidamente informado ${ }^{59}$. Por ello, considera Puppe con razón la pregunta por el consentimiento informado del paciente por principio como imposible de responder y, por consiguiente, como carente de sentido, pues no existe a esta pregunta ninguna respuesta verdadera ${ }^{60}$.

Kuhlen replica a este argumento que con frecuencia en la práctica jurídica y también en la vida cotidiana se formulan preguntas acerca de decisiones que en la realidad no fueron tomadas, sin que dichas preguntas sean consideradas como imposibles de responder, por cuanto la falta de leyes deterministas no impide que semejantes preguntas puedan ser respondidas con mayor o menor verosimilitud ${ }^{61}$.

Frente a este contraargumento, sin embargo, debe recalcarse que solamente puede obtenerse una respuesta verosímil a la pregunta de cómo alguien se hubiera comportado en una situación hipotética según el criterio de un hombre medio razonable. Esta solución, sin embargo, difícilmente puede resultar compatible con la tesis de la jurisprudencia ${ }^{62}$ y de

\footnotetext{
57 Puppe (2003) 768 ss.; Puppe, Ingeborg (2004). "Hypothetische Einwilligung bei medizinischem Heileingriff”. JR 2004, pp 469-472, p. 470; Puppe (2011) \$11/ NM 19 ss. En el mismo sentido Otto (2004) 683; Sternberg-Lieben, Detlev (2008). Anmerkung zu BGH StV 2008, 189. StV 2008, pp. 190-193, p. 192.

58 Puppe (2003) 769; Puppe (2004) 470.

59 Puppe (2004) 470; SChneider, Hendrik (2009). "Bleibendes im arzstrafrechtlichen Denken Eberhard Schmidts". En Festschrift der Juristenfakultät zum 600jährigen Bestehen der Universität Leipzig (editado por miembros de la Facultad de Derecho de la Universidad de Leipzig). Berlin: Duncker \& Humblot, pp. 165-180, p. 178.

$60 \quad$ Puppe (2003) 769; Puppe (2004) 470.

61 Kuhlen (2004) 228. En términos similares Roxin (2006) \$13 / NM 133.

62 BGH JR 2004, 715.
} 
los partidarios del consentimiento hipotético en la doctrina ${ }^{63}$ en el sentido que esta figura no atiende a si una persona razonable hubiera consentido en la intervención, sino exclusivamente a si el afectado concreto hubiera prestado o no su consentimiento. Muchos pacientes no toman sus decisiones necesariamente como un hombre racional ficticio, sino sobre la base de aspectos dominados por el sentimiento e incluso por un elemento irracional, lo cual, como con acierto lo ha destacado Paeffgen, pertenece al ámbito propio de la responsabilidad individual en una sociedad libre ${ }^{64}$.

La sugerencia de la jurisprudencia ${ }^{65}$ y de algunos partidarios del consentimiento hipotético ${ }^{66}$ de determinar la decisión hipotética del titular del bien jurídico mediante la consulta al paciente luego de la intervención resulta además difícilmente practicable. De aceptarse este método, se pondría la punibilidad del médico a disposición de la víctima, ${ }^{67}$ lo cual conllevaría enormes problemas. En primer lugar, el peligro de que el paciente pudiera tener en sus manos la posibilidad de "vender" su declaración sobre su consentimiento hipotético no puede descartarse ${ }^{68}$. Escuchar al paciente resulta además imposible cuando este ha fallecido como consecuencia del procedimiento médico ${ }^{69}$. En pacientes comatosos ello sería posible, en el supuesto de que exista una recuperación, solamente luego de semanas, meses o incluso años ${ }^{70}$.

\section{2) SUPRESIÓN DE LA AUTONOMÍA DEL PACIENTE MEDIANTE LA APLICACIÓN IMPROCEDENTE DEL PRINCIPIO IN DUBIO PRO REO}

Según el BGH, de permanecer dudas acerca del consentimiento hipotético del titular del bien jurídico, se debe asumir a favor del médico que el paciente hubiera consentido en la intervención en caso de haber

63 Kuhlen (2005) 715.

64 Paeffgen, Hans-Ullrich (2004). "Gefahr-Definition, Gefahr-Verringerung und Einwilligung im medizinischen Bereich. Problem Beschreibung und Methodologisches, veranschaulicht am Beispiel des virus-infektiösen Chrirurgen". En Rogall, Klaus et al. (coordinadores): Festschrift für Hans-Joachim Rudolphi zum 70. Geburtstag. Neuwied: Luchterhand, pp. 187-211, p. 209.

65 BGH JR 2004, 252.

66 Roxin (2006) \$13 / NM 127.

67 JäGER, Christian (2007). "Die hypothetische Einwilligung - ein Fall der rückwirkenden juristischen Heilung in der Medizin". En Müller-Dietz, Hans et al. (coordinadores): Festschrift für Heike Jung zum 65. Geburtstag am 23. April 2007. Baden-Baden: Nomos, pp. 345-360, p. 354. En similares términos Schneider (2009) 178.

68 Gropp, Walter (2006). "Hypothetische Einwilligung im Strafrecht". En Hoyer, Andreas et al. (coordinadores): Festschrift für Friedrich-Christian Schroeder zum 70. Geburtstag. Heidelberg: C.F. Müller, pp. 197-207, p. 200.

69 Paeffgen (2004) 208; Sternberg-Lieben (2008) 192; Schneider (2009) 178; Sowada, Christoph (2012). "Die Hypothetische Einwilligung im Strafrecht". NStZ 2012, pp. 1-10, p. 6.

70 JÄGER (2007) 355. 
sido debidamente informado ${ }^{71}$. Esta aplicación del principio pro reo resulta problemática, como se demuestra a continuación.

Como con razón advierte Puppe, mediante esta aplicación del señalado principio podría el médico obligar al paciente mediante información incompleta o falsa a soportar todo tratamiento médico que se mantenga dentro del marco de la lex artis, toda vez que la prueba de que el titular del bien jurídico no hubiera consentido en la intervención resulta siempre imposible en tanto exista una indicación médica para la operación ${ }^{72}$. Con ello, importa esta aplicación del principio pro reo en los casos de consentimiento hipotético una supresión indeseable desde un punto de vista político-criminal de la autonomía del paciente, la cual es reemplazada por criterios de verosimilitud ${ }^{73}$. Este es por sobre todo el caso de aquellos partidarios del consentimiento hipotético en la doctrina que se manifiestan expresamente a favor de una determinación del contenido de dicho consentimiento hipotético del paciente mediante criterios de verosimilitud o mediante máximas de la experiencia extraídas del campo de la psicología $^{74}$.

La aplicabilidad misma del principio in dubio pro reo en casos de consentimiento hipotético es altamente dudosa. Dicho principio resulta aplicable solamente respecto de un hecho, ${ }^{75}$ y no respecto de una mera presunción (es decir, en el caso de procesos enteramente indeterminados), como es el caso de la pregunta acerca de cómo hubiera decidido alguien en caso de haber sido informado debidamente y de antemano acerca de la intervención ${ }^{76}$.

La propuesta de Roxin ${ }^{77}$ de limitar la aplicación del principio in $d u$ bio pro reo en el ámbito del consentimiento hipotético mediante la teoría del incremento del riesgo no resulta tampoco convincente. Sin entrar en

71 BGH NStZ 1996, 35; BGH JR 2004, 252.

72 Puppe (2004) 471; Puppe (2003) 769; Sowada (2012) 7.

73 Puppe (2003) 769. En similares términos Duttge, Gunnar (2006). "Die hypothetische Einwilligung als Strafsauschlußgrund: wegweisende Innovation oder Irrweg?”. En Hoyer, Andreas et al. (coordinadores): Festschrift für Friedrich-Christian Schroeder zum 70. Geburtstag. Heidelberg: C.F. Müller, pp. 179-195, p. 188; Bollacher / Stockburger (2006) 913; Weber, Ulrich (2011). "Zu den Grenzen des strafrechtlichen Denkens in RechtsmäBigkeitsalternativen”. En Paeffgen, Hans-Ullrich et al. (coordinadores): Strafrechtswissenschaft als Analyse und Konstruktion. Festschrift für Ingeborg Puppe zum 70. Geburtstag. Berlin: Duncker \& Humblot, pp. 1059-1065, p. 1064.

74 Como es por ejemplo el caso de Rönnau (2004) 802.

75 KindhäUSER (2010) \$23 / NM 69.

76 Paeffgen (2004) 208. En igual sentido Duttge (2006) 189; Sowada (2012) 7. Véase también Schlüchter, Ellen (2007). \$261. En Rudolphi, Hans-Joachim et al.: Systematischer Kommentar zur Strafprozessordnung und zum Gerichtsverfassungsgesetz. 40 Tomo. München: Luchterhand, NM 73; SchlüChter, Ellen (1984). Anmerkung zu BGH JR 1984, 514. JR 1984, 517-522, p. 520; Puppe, Ingeborg (1994). Anmerkung zu BGH JR 1994, 514. JR, pp. 515-518, p. 517.

Véase nota 45. 
la naturaleza debatida de la teoría del incremento del riesgo como tal ${ }^{78}$, la determinación acerca de en cuáles casos el paciente con seguridad hubiera prestado su consentimiento de haber sido informado debidamente resulta imposible, como ya se ha demostrado ${ }^{79}$.

\section{3) Dificultades de la SANCión a título de TENTATiva EN loS CASOS DE CONSENTIMIENTO HIPOTÉTICO}

Con la sanción de los supuestos de consentimiento hipotético a título de tentativa tienen los partidarios de dicha figura como fin evitar la afectación del derecho de autodeterminación del paciente ${ }^{80}$. Esta solución, sin embargo, protegería la autonomía del paciente de un modo meramente aparente, en cuanto una responsabilidad a título de tentativa no se presentaría nunca o, a lo sumo, en un número muy reducido de casos.

Cuando se asume que la imputación del resultado sería siempre imposible debido a la aplicación del principio in dubio pro reo $o^{81}$, no se presentaría nunca una punibilidad del médico a título de tentativa cuando el médico supiera esto, pues carecería de la voluntad de causar el resultado de modo imputable y con ello faltaría el dolo de consumación necesario para hacer nacer una responsabilidad penal del médico a título de tentati$\mathrm{va}^{82}$.

$\mathrm{Si}$, por el contrario, se considera junto con Kuhlen como posible la determinación del consentimiento hipotético de la víctima, podría afirmarse una punibilidad a título de tentativa solamente cuando el agente parte del supuesto que el titular del bien jurídico no hubiera consentido en la intervención ${ }^{83}$. Las dificultades relativas a la prueba de semejante representación del médico son considerables; todo médico podría señalar en su defensa que asumió que el paciente hubiera prestado su consentimiento de haber sido debidamente informado ${ }^{84}$.

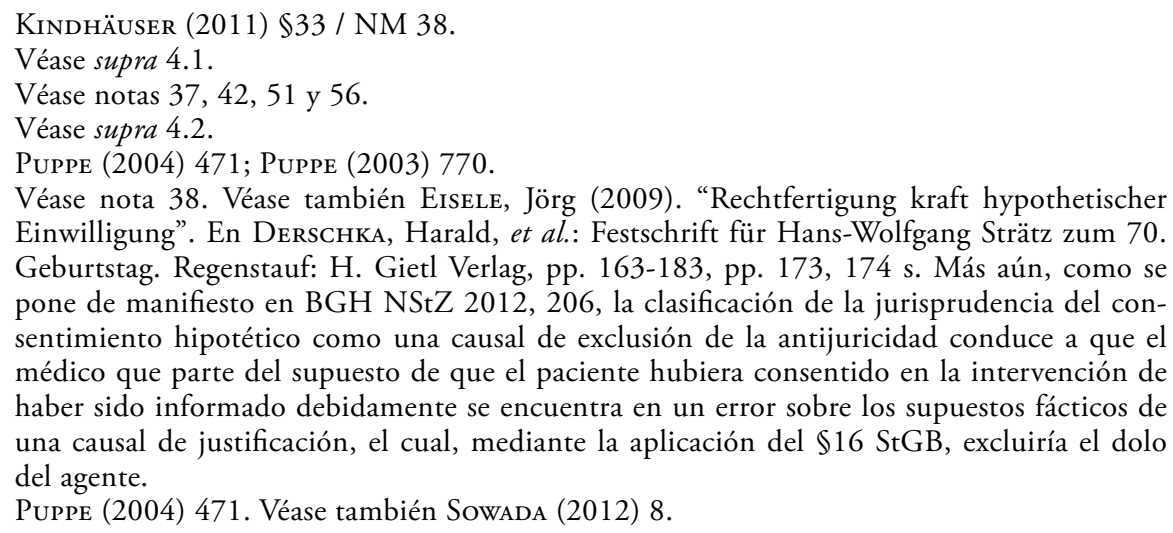
Einwilligung". En DerschKa, Harald, et al.: Festschrift für Hans-Wolfgang Strätz zum 70. Geburtstag. Regenstauf: H. Gietl Verlag, pp. 163-183, pp. 173, 174 s. Más aún, como se pone de manifiesto en BGH NStZ 2012, 206, la clasificación de la jurisprudencia del consentimiento hipotético como una causal de exclusión de la antijuricidad conduce a que el médico que parte del supuesto de que el paciente hubiera consentido en la intervención de haber sido informado debidamente se encuentra en un error sobre los supuestos fácticos de una causal de justificación, el cual, mediante la aplicación del $\$ 16 \mathrm{StGB}$, excluiría el dolo del agente. 


\section{4) RELATIVIZACIÓN DE LOS REQUISITOS DEL CONSENTIMIENTO PRESUNTO - RELACIÓN CON EL CONSENTIMIENTO EFECTIVO}

El reconocimiento de la figura del consentimiento hipotético podría conducir a que el consentimiento presunto de la víctima como causal de justificación se vuelva superfluo. Los estrictos requisitos del consentimiento presunto, que deben determinarse según un criterio ex ante, perderían su sentido si la consideración ex post del consentimiento hipotético de la víctima pudiera tener como resultado la exclusión del injusto de un delito consumado ${ }^{85}$. En contra de Kuhlen ${ }^{86}$, una punibilidad a título de tentativa no evitaría este peligro, por cuanto, según lo hemos demostrado, la posibilidad de afirmar una tentativa en los supuestos de consentimiento hipotético resulta cuando menos dudosa ${ }^{87}$.

Además de lo anterior, en la relación entre el consentimiento efectivo de la víctima y el consentimiento hipotético permanece sin aclarar por qué los vicios de la voluntad son relevantes en la prueba del consentimiento efectivo, mientras que en el consentimiento hipotético se les trata como irrelevantes ${ }^{88}$.

\section{5) INCONSISTENCIAS DE UN TRASLADO DE LOS CRITERIOS DE IM- PUTACIÓN OBJETIVA AL PLANO DE LA ANTIJURICIDAD}

La fundamentación de Kuhlen de la figura del consentimiento hipotético mediante el desplazamiento de los criterios de la imputación objetiva al plano de la antijuricidad resulta dogmáticamente poco convincente. Diversos argumentos justifican este juicio sobre la doctrina de Kuhlen, los que a continuación se analizan.

85 Bollacher / Stockburger (2006) 913; Eisele (2005) 254; Eisele (2009) 173; Eser, Albin / Sternberg-Lieben, Detlev (2010). \$223. En Schönke, Adolf / Schroeder, Horst / EsEr, Albin (coordinadores): Strafgesetzbuch Kommentar. 28 a edición. München: Beck, NM 40e. Por su parte, Schlehofer, Horst (2011). “"Pflichtwidrigkeit” und "Pflichwidrigkeitszusammenhang” als Rechtswidrigkeitsvoraussetzungen?”. En PAeffgen, Hans-Ullrich et al. (coordinadores): Strafrechtswissenschaft als Analyse und Konstruktion. Festschrift für Ingeborg Puppe zum 70. Geburtstag. Berlin: Duncker \& Humblot, pp. 953-970, pp. 965 s., llama la atención sobre la dificultad relativa al hecho de que, a diferencia de lo que ocurre en el consentimiento presunto, en el consentimiento hipotético no existe un interés preponderante ex ante que pueda justificar la exclusión del injusto de la conducta, como lo ha pretendido la jurisprudencia del BGH.

$86 \quad$ Véase nota 37.

$87 \quad$ Véase supra 4.3.

88 SiскоR, Jens Andreas (2008). "Logische Unstimmigkeiten in der höchstrichterlichen Prüfungsformel zur hypothetischen Einwilligung”. JR 2008, pp. 179-185, p. 182 [en adelante, citado como Sickor (2008b)]; Eser / STERnbERG-Lieben (2010) \$263 / NM 40e. 


\subsection{1) ¿Un nexo causal en las causales de justificación?}

Según Kuhlen, debe probarse en el plano de la antijuricidad la imputación objetiva del resultado típico al déficit en los requisitos de una causal de justificación en aquellos supuestos de consentimiento hipotético ${ }^{89}$.

Dicho del modo más elemental posible, la teoría de la imputación objetiva tiene en el plano de la tipicidad por principal función la tarea de determinar normativamente que el resultado típico se encuentra determinado por un proceso causal riesgoso y no permitido, bajo dominio del autor, a efecto de excluir la responsabilidad penal en aquellos casos de procesos causales penalmente irrelevantes ${ }^{90}$.

Semejante función no podría ser cumplida por la imputación objetiva en el plano de la antijuricidad ya desde un primer principio, por cuanto entre el cumplimiento o incumplimiento de los requisitos de una causal de justificación, por una parte, y el resultado típico, por otra, no existe ni puede existir vínculo causal alguno ${ }^{91}$. Con otras palabras, los requisitos de una causal de justificación no causan el resultado, sino que solamente excluyen el injusto de dicho resultado ${ }^{92}$. Según lo formula Duttge, el desplazamiento de los criterios de imputación objetiva al plano de la antijuricidad importa un desconocimiento de la naturaleza propia de esta, pues en este nivel no se afirma el "injusto total" mediante elementos adicionales que fundamenten el injusto, sino solamente la falta de los requisitos de alguna causal de justificación ${ }^{93}$. Consecuentemente, consiste la falla metodológica de la fundamentación de Kuhlen del consentimiento hipotético en que implícitamente se busca un nexo causal entre el déficit de justificación y el resultado típico ${ }^{94}$.

\subsection{2) El consentimiento hipotético como una causa de reemplazo penalmente irrelevante}

En los casos de consentimiento hipotético se ha hecho cierto que el titular del bien jurídico en la realidad no ha prestado un consentimiento

89 Véase nota 24.

90 KINDHÄUSER $(2011) \$ 11 /$ NM 2.

91 Puppe (2003) 770; Duttge (2006) 185 ss. Véase también, desde la perspectiva comparada del derecho japonés, YamanaKa, Keiichi (2010): "Kritisch-dogmatische Überlegungen zur hypothetischen Einwilligung". En Bloy, René, et al. (coordinadores): Gerechte Strafe und legitimes Strafrecht, Festschrift für Manfred Maiwald zum 75. Geburtstag. Berlin: Duncker \& Humblot, pp. 865-884, p. 880. 191; BöCKer, Phillip (2005). "Die hypothetische Einwilligung im Zivil- und Strafrecht. JZ 2005, pp. $925-932$, p. 930 .

93 Duttge (2006) 186. En similares términos Eisele (2009) 174; Sowada (2012) 9.

94 Puppe (2003) 770. Otra opinión Kuhlen (2004) 228, quien rechaza una comprensión causal de su tesis. 
válido respecto de la intervención curativa. Con ello, es el consentimiento hipotético de la víctima en el tratamiento médico nada más que una causa de reemplazo ${ }^{95}$. Según una visión ya asentada en la doctrina, las llamadas causas de reemplazo han de considerarse como penalmente irrelevantes ${ }^{96}$.

El que la lesión de un bien jurídico en razón de un curso causal hipotético no se hubiera producido no se le asigna en el derecho penal importancia alguna. De lo contrario, perdería un bien jurídico amenazado por una pronta lesión su función de garantía normativa ${ }^{97}$. Ello es válido también para aquellos casos en que el resultado de todos modos se hubiera producido por otro comportamiento de la víctima ${ }^{98}$. Respecto de la pregunta por el comportamiento alternativo conforme a derecho, se trata exclusivamente del reemplazo de la conducta del agente contraria a deber por una conducta ajustada a Derecho; la conducta de la víctima, en cambio, debe valorarse exclusivamente del modo en que efectivamente ocurrió99.

En otras palabras, un bien jurídico protegido no pierde su pretensión de ser respetado en el momento de su lesión si el titular de dicho bien jurídico con posterioridad hubiera renunciado a la protección del ordenamiento jurídico ${ }^{100}$. En el caso del hurto nadie pondría en duda la punibilidad del ladrón si el propietario de la cosa hurtada con posterioridad a la consumación del delito afirmara que le hubiera regalado el objeto al autor de habérselo pedido este ${ }^{101}$.

\subsection{3) Inexistencia de una "analogía estructural" entre delitos de omisión e imprudentes y el consentimiento hipotético}

En contra de la idea de considerar al consentimiento hipotético de la víctima como una causa de reemplazo irrelevante podría argumentarse que en otros ámbitos la introducción de dichos cursos causales hipotéticos se encuentra permitida, a saber en los delitos de omisión impropia

\footnotetext{
95 Puppe (2004) 471; Отto (2004) 683.

96 KindhäUser (2011) \$10 / NM 18 y ss.

97 Puppe (2003) 768.

98 JäGER (2007) 353. Sorprendentemente afirma Roxin (2006) \$11 / NM 60, partidario del consentimiento hipotético, lo siguiente: "Quien, por consiguiente, derriba un árbol ajeno, que el propietario mismo deseaba derribar (...) sigue siendo responsable por el delito de daños".

99 Jäger (2007) 353; Eser / Sternberg-Lieben (2010), \$223, NM 40e. En términos similares Weber (2011) 1064; SoWAdA (2012) 10.

100 Отто (2004) 683. Véase también Otтo, Harro / Albrecht, Andreas (2010): “Die Bedeutung der hypothetischen Einwilligung für den ärztlichen Heileingriff”. Jura 2010, pp. 264271, p. 267 s.

101 Ejemplo en Отто (2004) 683; Eisele (2005) 254; Duttge (2006) 188.
} 
y en los delitos imprudentes ${ }^{102}$. En el caso de los delitos de omisión impropia la fórmula de la conditio sine qua non se ve alterada: la omisión se considera como causal cuando la acción omitida no puede ser agregada mentalmente sin que el resultado resulte suprimido ${ }^{103}$. En el caso de los delitos imprudentes se prueba respecto del nexo de infracción de un deber si la agregación de la acción ajustada al deber del agente con una probabilidad rayana en la certeza hubiera evitado el resultado típico ${ }^{104}$.

Sin embargo, no existe en primer lugar ningún paralelo entre delitos de omisión impropios y el consentimiento hipotético. En esta última figura se omite la entrega de la información debida, pero esta omisión se encuentra en un plano previo temporalmente a la intervención y permanece como irrelevante cuando dicha intervención -mediante un comportamiento activo- no se lleva a cabo ${ }^{105}$.

La existencia de una analogía entre el nexo de infracción de un deber en los delitos imprudentes y el consentimiento hipotético, un elemento esencial de la doctrina de Kuhlen, debe descartarse igualmente. En los casos de consentimiento hipotético por regla general se lleva a cabo la intervención de modo doloso, por cuanto el médico de ordinario actúa en el entendido que no concurre el consentimiento del paciente. Ello implica que el autor no se encuentra en un error sobre los supuestos fácticos de una causal de justificación que excluya el dolo y deje subsistente la culpa $^{106}$. La figura del nexo de infracción de un deber, sin embargo, pertenece a la dogmática del delito imprudente y no ha encontrado hasta el momento aceptación en el ámbito del delito doloso ${ }^{107}$.

\section{6) IMPROCEDENCIA DE LA EXCLUSIÓN DE LA RESPONSABILIDAD PENAL EN VIRTUD DE “FRAGMENTOS DE UNA JUSTIFICANTE”}

La fundamentación de Mitsch del consentimiento hipotético mediante lo que este autor llama "fragmentos de justificante"108 debe rechazarse de plano. Los elementos de una causal de justificación deben concu-

102 Gropp (2006) 202.

103 Baumann, Jürgen / Weber, Ulrich / Mitsch, Wolfgang (2003). Strafrecht Allgemeiner Teil. $11^{\text {a }}$ edición. Bielefeld: Gieseking, $\$ 15$ / NM 24. No cabe tratar aquí el problema de si puede hablarse stricto sensu de causalidad en la omisión.

104 KindhäUSER (2011) \$33 / NM 38.

105 Gropp (2006) 202. En igual sentido Otto / Albrecht (2010) 268.

106 Gropp (2006) 202.

107 Gropp (2006) 202. Según Gropp, el nexo de infracción de un deber podría ser visto como un correctivo de la formulación típica amplia propia del delito imprudente. Este argumento no se adapta al caso del delito doloso y por lo tanto no podría justificar la aplicación de este correctivo. Sobre la falta de un paralelo entre la constelación de la falta del nexo de infracción de deber y el consentimiento hipotético véase también Otto / Albrecht (2010) 269; Schlehofer (2011) 969 s.; Yamanaka (2010) 881. 
rrir en la realidad y no de un modo meramente hipotético para surtir un efecto justificante de la conducta ${ }^{109}$. Los elementos de una justificante o concurren íntegramente o bien no se cumplen. La punibilidad a título de delito consumado no puede excluirse con el argumento de que los requisitos de una causal de justificación se cumplen parcialmente ${ }^{110}$.

\section{CONCLUSIONES}

En razón de los argumentos desarrollados, la aplicación en materia penal de la figura del consentimiento hipotético, creada por la jurisprudencia del $\mathrm{BGH}$, debe ser rechazada. La determinación del consentimiento hipotético resulta imposible por principio, por cuanto no existen leyes naturales a nuestra disposición que permitan semejante constatación. Asimismo, importa el reconocimiento del consentimiento hipotético una afectación considerable de la autonomía del paciente, la que sería reemplazada por criterios de verosimilitud. El consentimiento presunto se encontraría además en peligro de desaparecer de reconocérsele efectos excluyentes de la responsabilidad penal al consentimiento hipotético determinado ex post.

Poco convincente dogmáticamente resulta la fundamentación de esta figura por algunos autores mediante un desplazamiento de los criterios de la imputación objetiva al plano de la antijuricidad, por cuanto no existe un vínculo causal entre la falta de cumplimiento del deber de informar y el resultado típico. Resulta además extremadamente dudoso que pueda dársele aplicación al correctivo del nexo de infracción de un deber en el ámbito del consentimiento hipotético, como lo postula Kuhlen ${ }^{111}$. Por el contrario, el consentimiento hipotético no puede ser tratado más que como una causa de reemplazo penalmente irrelevante.

Mediante la aplicación restringida de esta figura en la jurisprudencia alemana, exclusivamente en el ámbito de las intervenciones curativas médicas se ha creado asimismo un privilegio para la profesión médica cuyos fundamentos no pueden advertirse ${ }^{112}$. Resulta además altamente dudoso que la aplicación por parte del BGH del consentimiento hipotético también en casos de consentimiento del paciente obtenido mediante engaño sea deseable desde un punto de vista político-criminal ${ }^{113}$.

\footnotetext{
109 Duttge (2006) 187, nota 71.

110 Ello con mayor razón en aquellos ordenamientos como el chileno, donde al cumplimiento parcial de los requisitos de una causal de justificación se le atribuye un efecto meramente atenuante (Art. $11 \mathrm{~N}^{\circ} .1 \mathrm{CP}$ ).

111 Véase nota 24.

112 SICKOR (2008a) 16.

113 SICKOR (2008a) 16.
} 
Idénticos argumentos a los aquí expuestos son válidos para rechazar la aplicación de la figura del consentimiento hipotético también en el ordenamiento jurídico chileno de llegar a plantearse, pues, hasta este momento, no ha recibido la atención de la jurisprudencia ni de la doctrina nacional, la que se ha centrado en el problema previo de la necesidad de un consentimiento informado del paciente en el ámbito de las intervenciones médicas con propósito curativo, sus requisitos y efectos en cuanto a la eventual responsabilidad penal del médico ${ }^{114}$. Excede del propósito de este trabajo tomar una postura fundada sobre el consentimiento informado en el ámbito médico y su significado e implicancias jurídicopenales. Sin embargo, debe constatarse como una realidad la creciente importancia que ha cobrado este elemento para ciertos autores en la doctrina penal nacional reciente ${ }^{115}$ para los efectos de otorgar legitimidad a la intervención médica curativa practicada según la lex artis y hacerla así penalmente irrelevante. Dicha tendencia hará inevitable pronunciarse también acerca del consentimiento hipotético como posible correctivo para eventuales consecuencias indeseables que se sigan de la introducción del consentimiento informado en el ámbito penal.

Es a esta discusión que este trabajo ha intentado contribuir.

\section{BibLIOGRAFÍA}

- Baumann, Jürgen / Weber, Ulrich / Mitsch, Wolfgang (2003). Strafrecht, Allgemeiner Teil Lehrbuch. $11^{\text {a }}$ edición. Bielefeld: Gieseking.

- Böcker, Philipp (2005). "Die hypothetische Einwilligung im Zivilund Strafrecht". JZ 2005, pp. 925-932.

- Bollacher, Florian / Stockburger, Jochen (2006). "Der ärztliche Heileingriff in der strafrechtlichen Fallbearbeitung”. Jura 2006, pp. 908-914.

- Bosch, Nikolaus (2008). "Grenzen einer hypothetischen Einwilligung bei ärztlichem Eingriff”. JA 2008, pp. 70-72.

- Duttge, Gunnar (2006). "Die hypothetische Einwilligung als Strafausschlußgrund: wegweisende Innovation oder Irrweg?”. En

114 Para una visión de conjunto sobre el consentimiento informado del paciente en el ámbito penal chileno, con énfasis en el análisis sobre las diversas posturas posibles sobre este punto y sus consecuencias dogmáticas y prácticas, véase Hernández, Héctor (2010). "Consentimiento informado y responsabilidad penal médica: una relación ambigua y problemática”. Cuadernos de Análisis Jurídico, Colección Derecho Privado (Universidad Diego Portales). N. ${ }^{\circ}$ 6, pp. 167-185.

115 Véase Hernández (2010) 173, con referencias; Mayer, Laura (2011). "Autonomía del paciente y responsabilidad penal médica". Revista de Derecho de la Pontificia Universidad Católica de Valparaíso. N. ${ }^{\circ}$ XXVII, pp. 371-413. 
Hoyer, Andreas et al. (coordinadores): Festschrift für FriedrichChristian Schroeder zum 70. Geburtstag. Heidelberg: C.F. Müller, pp. 179-195.

- Eisele, Jörg (2005). "Hypothetische Einwilligung bei ärztlichen Aufklärungfehlern”. JA 2005, pp. 252-254.

(2009). "Rechtfertigung kraft hypothetischer

Einwilligung”. En Derschka, Harald, et al.: Festschrift für HansWolfgang Strätz zum 70. Geburtstag. Regenstauf: H. Gietl Verlag, pp. 163-183.

- Eser, Albin / Sternberg-Lieben, Detlev (2010). \$223. En Schonke, Adolf / Schröder, Horst / Eser, Albin et al. (coordinadores): Strafgesetzbuch Kommentar. 28a edición. München: Beck.

- Gropp, Walter (2006). "Hypothetische Einwilligung im Strafrecht". En Hoyer, Andreas et al. (coordinadores): Festschrift für FriedrichChristian Schroeder zum 70. Geburtstag. Heidelberg: C.F. Müller, pp. 197-207.

- Hernández, Héctor (2010). "Consentimiento informado y responsabilidad penal médica: una relación ambigua y problemática". Cuadernos de Análisis Jurídico, Colección Derecho Privado (Universidad Diego Portales). N. ${ }^{\circ}$ 6, pp. 167-185.

- JäGER, Christian (2007). "Die hypothetische Einwilligung - ein Fall der rückwirkenden juristischen Heilung in der Medizin”. En MüllerDietz, Heinz et al. (coordinadores): Festschrift für Heike Jung zum 65. Geburtstag am 23. April 2007. Baden-Baden: Nomos, pp. 345360.

- KindhäUser, Urs (2010). Strafprozessrecht. 2a edición. BadenBaden: Nomos.

Baden-Baden: Nomos.

(2011). Strafrecht Allgemeiner Teil. 5a edición.

(2012). Strafrecht Besonderer Teil I. 5a edición.

Baden-Baden: Nomos.

- Kuhlen, Lothar (2001). "Objektive Zurechnung bei Rechtfertigungsgründen”. En Schünemann, Bernd et al. (coordinadores): Festschrift für Claus Roxin zum 70. Geburtstag am 15. Mai 2001. Berlin-New York: De Gruyter, 331-347. [citado como Kuhlen (2001a)].

bei Mängeln der wirklichen und der mutmaßlichen Einwilligung". En Britz, Guido et al. (coordinadores): Grundfragen staatlichen Strafens. Festschrift für Heinz Müller-Dietz zum 70. Geburtstag. München: Beck, pp. 431-451. [citado como Kuhlen (2001b)]. 
(2004). "Ausschluss der objektiven Erfolgszurechnung bei hypothetischer Einwilligung des Betroffenen”. JR 2004, pp. 227230.

Erfolgsrechtfertigung". JZ 2005, pp. 713-718.

(2005). "Hypothetische Einwilligung und

- MaYer, Laura (2011). "Autonomía del paciente y responsabilidad penal médica". Revista de Derecho de la Pontificia Universidad Católica de Valparaíso. N. ${ }^{\circ}$ XXVII, pp. 371-413.

- Mitsch, Wolfgang (2005). "Die hypothetische Einwilligung im Arztstrafrecht”. JZ 2005, pp. 279-285.

- Отто, Harro (2004). "Einwilligung, mutmaßliche, gemutmaßte und hypothetische Einwilligung”. Jura 2004, pp. 679-683.

- Oтto, Harro / Albrecht, Andreas (2010). "Die Bedeutung der hypothetischen Einwilligung für den ärztlichen Heileingriff”. Jura 2004, pp. 264-271.

- Paeffaen, Hans-Ullrich (2004). "Gefahr-Definition, GefahrVerringerung und Einwilligung im medizinischen Bereich. Problem-Beschreibung und Methodologisches, veranschaulicht am Beispiel des virus-infektiösen Chirurgen”. En Rogall, Klaus et al. (coordinadores): Festschrift für Hans-Joachim Rudolphi zum 70. Geburtstag. Neuwied: Luchterhand, pp. 187-211.

- Puppe, Ingeborg (1994). Anmerkung zu BGH JR 1994, 514. JR 1994, pp. 515-518.

(2003). "Die Strafrechtliche Verantwortlichkeit des Arztes bei mangelnder Aufklärung über eine BehandlungsalternativeZugleich Besprechung von BGH, Urteile vom 3.3.1994 und 29.6.1995”. GA 2003, pp. 764-776.

(2004). "Hypotethische Einwilligung bei medizinischem Heileingriff. Urt. des BGH v. 20.1.2004- 1 StR 319/03”. JR 2004, pp. 469-472.

Rechtsprechung. $2^{\text {a }}$ edición. Baden-Baden: Nomos.

(2011). Strafrecht Allgemeiner Teil im Spiegel der

- Rigizahn, Ernest F. (1996). Anmerkung zu BGH JR 1996, 69. JR 1996, pp. 72-75.

- Rönnau, Thomas (2004). Anmerkung zu BGH JZ 2004, 800. JZ 2004, pp. 801-804.

- Rosenau, Henning (2010). "Die hypothetische Einwilligung im Strafrecht". En Bloy, René, et al. (coordinadores): Gerechte Strafe und legitimes Strafrecht, Festschrift für Manfred Maiwald zum 75. Geburtstag. Berlin: Duncker \& Humblot, pp. 683-700.

- Roxin, Claus: Strafrecht Allgemeiner Teil I: Grundlagen-Der Aufbau der Verbrechenslehre. 4a edición. München: Beck. 
- Schlehofer, Horst (2011). "Pflichtwidrigkeit' und 'Pflichwidrigkeitszusammenhang' als Rechtswidrigkeitsvoraussetzungen?”. En Paeffgen, Hans-Ullrich et al. (editores): Strafrechtswissenschaft als Analyse und Konstruktion. Festschrift für Ingeborg Puppe zum 70. Geburtstag. Berlin: Duncker \& Humblot, pp. 953-970.

- Schlüchter, Ellen (2007). \$261. En Rudolphi, Hans-Joachim et al.: Systematischer Kommentar zur Strafprozessordnung und zum Gerichtsverfassungsgesetz. 4o Tomo. (\$\$198-263). München: Luchterhand.

1984, pp. 517-522.

(1984). Anmerkung zu BGH JR 1984, 514. JR

- Schneider, Hendrik (2009). "Bleibendes im arzstrafrechtlichen Denken Eberhard Schmidts". En Festschrift der Juristenfakultät zum 600jährigen Bestehen der Universität Leipzig (editado por miembros de la Facultad de Derecho de la Universidad de Leipzig). Berlin: Duncker \& Humblot, pp. 165-180.

- Sickor, Jens Andreas (2008). "Die Übertragung der hypothetischen Einwilligung auf das Strafrecht”. JA 2008, pp. 11-16 [citado como Sickor (2008a)].

- (2008). "Logische Unstimmigkeiten in der höchstrichterlichen Prüfungsformel zur hypothetischen Einwilligung". JR 2008, pp. 179-185 [citado como Sickor (2008b)].

- Sowada, Christoph (2012). "Die Hypothetische Einwilligung im Strafrecht”. NStZ 2012, pp. 1-10.

- Sternberg-Lieben, Detlev (2008). Anmerkung zu BGH StV 2008, 189. StV 2008, pp. 190-193.

- Tröndle, Herbert (1983). "Selbstbestimmung des Patienten Wohltat und Plage?”. MDR 1983, pp. 881-887.

- Ulsenheimer, Klaus: Anmerkung zu BGH NStZ 1996, 34. NStZ 1996, pp. 132-133.

- Weber, Ulrich (2011). "Zu den Grenzen des strafrechtlichen Denkens in Rechtsmäßigkeitsalternativen”. En Paeffgen, HansUllrich et al. (coordinadores): Strafrechtswissenschaft als Analyse und Konstruktion. Festschrift für Ingeborg Puppe zum 70. Geburtstag. Berlin: Duncker \& Humblot, pp. 1059-1065.

- YamanaKa, Keiichi (2010): "Kritisch-dogmatische Überlegungen zur hypothetischen Einwilligung". En Bloy, René, et al. (coordinadores): Gerechte Strafe und legitimes Strafrecht, Festschrift für Manfred Maiwald zum 75. Geburtstag. Berlin: Duncker \& Humblot, pp. 865-884. 


\section{JURISPRUDENCIA CITADA}

- $\quad$ RG. 31 de mayo de 1894. 3 Str 1406/94 (=RGSt 25, 375).

- BGH. 25 de septiembre de 1990. 5 StR 342/90 (=BGHR StGB $\$ 223$ I Heileingriff $2,1,3)$.

- $\quad$ BGH. 29 de junio de 1995. 4 StR 760/94 (=BGH NStZ 1996, 34; BGH JR 1996, 69; StV 1996, 148.

- $\quad$ BGH. 15 de octubre de 2003. 1 StR 300/03 (=BGH JR 2004, 251; BGH NStZ-RR 2004, 16; BGH JZ 2004, 800).

- $\quad$ BGH. 20 de enero de 2004. 1 StR 319/03 (=BGH NStZ 2004, 442; BGH JR 2004, 469).

- $\quad$ BGH. 5 de julio de 2007. 4 StR 549/06 (=NStZ-RR 2007, 340; BGH StV 2008, 189).

- $\quad$ BGH. 11 de octubre de 2011. 1 StR 134/11 (= NStZ 2012, 205).

\section{LISTADO DE ABREVIATURAS}

Art. artículo

BGH Bundesgerichtshof (Tribunal Supremo Federal de Alemania)

BGHR Rechtsprechung des BGH in Strafsachen (colección de jurisprudencia)

CP Código Penal (Chile)

GA Goltdammer's Archiv für Strafrecht (Revista)

JA Juristische Arbeitsblätter (Revista)

JR Juristische Rundschau (Revista)

Jura Juristische Ausbildung (Revista)

JZ Juristenzeitung (Revista)

MDR Monatsschrift für Deutsches Recht (Revista)

NM número marginal

NStZ Neue Zeitschrift für Strafrecht (Revista)

NStZ-RR NStZ-Rechtsprechungs-Report Strafrecht (Revista)

öStGB Código Penal de Austria

p.(pp.) página(s)

RG

Reichsgericht (Tribunal Supremo del Imperio Alemán)

RGSt

s.(ss.)

Sentencias del Reichsgericht (citadas por tomo y página).

StGB Strafgesetzbuch (Código Penal Alemán)

StPO Strafprozessordnung (Ley de Procedimiento Penal de Alemania)

StR Strafsenat (Cámara Penal)

$\mathrm{StV} \quad$ Strafverteidiger (Revista)

$\$(\$ \$) \quad$ parágrafo(s) 